Service social

\title{
La médiation familiale, par Lisette Laurent-Boyer, Cap-Saint-Ignace, Les Éditions Yvon Blais Inc., 1992, 223 pages.
}

\section{Madeleine Beaudry}

Volume 41, numéro 2, 1992

Bilan des réformes

URI : https://id.erudit.org/iderudit/706575ar

DOI : https://doi.org/10.7202/706575ar

Aller au sommaire du numéro

Éditeur(s)

École de service social de l'Université Laval

ISSN

1708-1734 (numérique)

Découvrir la revue

Citer ce compte rendu

Beaudry, M. (1992). Compte rendu de [La médiation familiale, par Lisette

Laurent-Boyer, Cap-Saint-Ignace, Les Éditions Yvon Blais Inc., 1992, 223 pages.]

Service social, 41(2), 165-167. https://doi.org/10.7202/706575ar d'utilisation que vous pouvez consulter en ligne.

https://apropos.erudit.org/fr/usagers/politique-dutilisation/ 


\section{LA MÉDIATION FAMILIALE}

collectif multidisciplinaire coordonné par LISETTE LAURENT-BOYER, Cap-Saint-Ignace, Les Éditions Yvon Blais inc., 1992, 223 pages.

Le premier livre écrit en français en Amérique du Nord sur la médiation familiale tombe à point. Près de la moitié des mariages se soldent par un divorce. Les hommes, les femmes et les enfants qui traversent cette période difficile et envisagent ses conséquences à long terme s'interrogent sur la meilleure manière de réorganiser leur vie et de régler leurs conflits. La médiation familiale propose une nouvelle approche pour aider ces personnes.

Dans cet ouvrage collectif et multidisciplinaire, huit travailleuses sociales et un travailleur social, une psychologue, trois avocats, dont une criminologueavocate et un juge, et enfin un notaire abordent plusieurs dimensions de la médiation familiale. En replaçant cette pratique dans son contexte historique et légal, ils en précisent la nature, clarifient le processus d'intervention, les connaissances et les habiletés particulières que doit maîtriser le médiateur. Ils examinent la place de l'enfant dans les décisions entourant la réorganisation familiale et font le point sur le partage des responsabilités parentales dans la garde conjointe des enfants après le divorce. Par ailleurs, ils abordent des thèmes plus généraux comme les aspects légaux et sociaux du divorce, de même que ses conséquences particulièrement pour les femmes et les enfants. Enfin, ils élargissent l'application du modèle de médiation au domaine de la protection de la jeunesse.

Chacun des quatorze auteurs aborde la médiation selon sa compétence, de sorte que l'ensemble de l'ouvrage reflète la pensée et la pratique multidisciplinaires dans ce domaine. Plutôt que de résumer chacun des articles du livre, il nous apparaît préférable d'en dégager les concepts de base.

Le code de déontologie de l'Association de médiation familiale du Québec définit la médiation familiale comme " une méthode de résolution de conflits basée sur la coopération, et par laquelle un tiers impartial et qualifié aide les membres de la famille à élaborer eux-mêmes une entente viable et satisfaisante pour chacun » (Laverdure, p. 85). Pour certains, la médiation naît d'une frustration à l'égard du système judiciaire qui, trop souvent, répond mal à la demande de citoyens pour un mode de résolution de conflits (McConomy, p. 148-149). Pour d'autres, elle s'inscrit dans une orientation de société qui encourage l'autodétermination, la communication, la responsabilité (Laurent-Boyer, p. 6). « Notre société moins paternaliste et moins moraliste invite les citoyens à discuter les services qu'on leur offre, qu'ils soient juridiques ou non juridiques, et à prendre eux-mêmes les décisions qui les concernent» (Lesage, p. 94).

Fidèle à ces valeurs, la médiation propose de créer un lieu de parole capable d'aider les personnes qui se séparent et leurs enfants à prendre eux-mêmes des décisions en fonction du meilleur intérêt de chacun, présent et futur. Pour ce faire, Françoise Lafortune présente un des modèles privilégiés par les médiateurs du Québec, celui de la " négociation sur intérêt ». Dans ce modèle, " les parties tentent de concilier leurs différends en examinant d'abord leurs intérêts communs et respectifs et seulement après, les différentes options se présentant à elles» (p. 46). Cette démarche n'est pas facile et les obstacles sont nombreux pour 
les conjoints. Dans un moment de grandes tensions où chacune des parties se sent menacée de tout perdre, le médiateur joue un rôle capital. Il doit suivre avec rigueur les étapes du processus d'intervention et maîtriser des habiletés particulières très bien décrites dans plusieurs chapitres du livre. Soulignons par exemple « I'habileté à aller chercher les informations pertinentes et à découvrir les besoins ou les intérêts sous-jacents de chacun, I'habileté à stimuler une discussion productive et à gérer l'interaction entre les parties » (Lafortune, p. 52).

De plus, ce livre aborde un enjeu majeur pour le médiateur : la participation des enfants dans le processus de médiation. Il suggère différentes modalités de participation de l'enfant (Baril, p. 181-190) et apporte un éclairage nouveau sur l'importance du maintien des responsabilités parentales par la garde partagée (Filion, p. 191-214). Après avoir relevé les avantages et les limites de cette modalité de garde, Filion conclut : « II est grand temps [...] que la loi soit modifiée pour reconnaître le droit des deux parents à prendre de façon égalitaire les décisions et le devoir de partager équitablement, selon leurs ressources et leurs différences, les responsabilités parentales » (p. 210).

Au-delà du conflit émotif, le divorce comporte une dimension légale qui situe la médiation familiale à " l'intersection de la relation d'aide et du droit » (Bérubé, p. 105-123). À cet égard, les auteurs insistent sur la complémentarité des formations en relations humaines et en sciences juridiques pour les médiateurs. $\mathrm{Si}$ « la pratique de la médiation exige une modification complète de la façon de penser de l'avocat» (Clairmont, p. 129), les travailleuses sociales et les psychologues « doivent apprendre à tenir compte du cadre juridique ainsi qu'à travailler au niveau des décisions concrètes des clients, que ce soit au niveau financier ou autre » (Bérubé, p. 117). Devant la complexité des connaissances et des habiletés particulières à la médiation, certains médiateurs travaillent en collaboration interprofessionnelle. "Le fait, pour des professionnels de disciplines différentes, de travailler ensemble a constitué un enrichissement inestimable et une occasion unique de développer des liens durables » (Bérubé, p. 120). Les auteurs ne manquent pas de mettre en lumière qu'il est temps d'orienter le débat sur le «comment intervenir ensemble » plutôt que sur le « à qui appartiendra la profession de la médiation » (Guilbault, p. 178).

La médiation est aussi un outil de prévention. Les conséquences psychologiques et économiques du divorce sur les enfants (présentées par Francine Guilbault, p. 161-166), le profond besoin qu'ils éprouvent de maintenir une relation significative avec leurs deux parents encouragent les médiateurs à aider les ex-conjoints à transformer leur relation de compétition en une relation de collaboration, à mettre l'accent sur la coresponsabilité parentale et le partage des ressources financières. C'est donc une forme d'éducation qui prépare les parents à faire face à leurs futures responsabilités parentales.

Ce livre rend bien compte de la compétence des auteurs, de leur expérience et de leurs connaissances dans le domaine de la médiation. Il a certes été dirigé par une main de maître, en s'assurant de la complémentarité des perspectives individuelles. Toutefois, certains thèmes se répètent d'un chapitre à l'autre : par exemple, I'historique, la philosophie, les définitions de la médiation. On souhaiterait alors plus d'unité, un regroupement logique plus serré de ces thèmes, ce qui éviterait les redondances ou faciliterait la compréhension des perspectives complémentaires. Mais en dépit de ces quelques lacunes, ce livre présente 
une bonne synthèse des connaissances sociales, juridiques et psychologiques dans le domaine de la médiation. Les personnes qui divorcent, tout comme les professionnels qui interviennent dans ce domaine et les étudiants, y trouveront une source de références précieuse, faisant le point sur cette pratique.

Madeleine BEAUDRY École de service social Université Laval

\section{LES VOIES NON VERBALES DU TRAVAIL SOCIAL}

Textes présentés au $\mathrm{Vl}$ e congrès du groupe régional européen de l'Association internationale des écoles de service social, publiés par l'École supérieure d'action sociale de Liège, Belgique, 1991, 204 pages.

Le titre de l'ouvrage m'avait amené à croire qu'il s'agissait d'une analyse des moyens et canaux non verbaux de la communication interpersonnelle et intergroupes. J'ai eu l'agréable surprise de découvrir un contenu fort différent, mais aussi intéressant que ce à quoi je $\mathrm{m}^{\prime}$ attendais. Dans la ligne de plusieurs approches européennes en travail social, Les voies non verbales du travail social présente des analyses théoriques et des expériences d'interventions sur les cadres de la vie quotidienne. Son titre indique une volonté de se démarquer des approches trop exclusivement verbales, empruntées à la psychothérapie et aux approches récentes en communication interpersonnelle. En même temps, la plupart des auteurs soulignent que la communication verbale en est augmentée et enrichie.

Présenté par le Dr Lopez, directeur de l'ESAS, I'ouvrage se compose de seize textes soumis au $\mathrm{Vl}^{\mathrm{e}}$ congrès de $\mathrm{l}^{\prime}$ Association internationale des écoles de service social par des praticiens-chercheurs et des professeurs de service social de sept pays. Les auteurs insistent sur l'organisation nouvelle et l'utilisation systématique des milieux de vie. Leur postulat est que le milieu de vie quotidienne constitue un cadre qui, au-delà des mots, développe une perception et un décodage qui lui sont propres. Ce cadre influence les échanges de valeurs, d'idées, de sentiments, et amène les personnes qui y vivent à développer de nouvelles significations des situations vécues.

Un groupe d'articles décrivent des expériences d'organisation de vie et d'activités, avec les groupes marginaux, les décrocheurs, les délinquants, les handicapés physiques et mentaux. Ils présentent des expériences d'appartements supervisés, de stage de travail ou de sport, d'intervention à domicile au lieu de cures de désintoxication. Casado souligne que même s'il y a de nombreux échanges verbaux avec les usagers, les principaux échanges se font avec les milieux, pour les amener à une organisation nouvelle et à un encadrement approprié. Dutrenit souligne qu'à l'établissement de fortes exigences, accompagnées de fortes récompenses, est habituellement reliée une amélioration sensible des comportements des usagers. 